\title{
A DATA MINING APPROACH FOR CUSTOMER SEGMENTATION USING A SAF-T BASED BUSINESS INTELLIGENCE SYSTEM
}

\author{
Rosa Silveira, Bruno Oliveira, Mariana Carvalho and Telmo Matos \\ ${ }^{I}$ CIICESI, Escola Superior de Tecnologia e Gestão, Politécnico do Porto, Portugal
}

\begin{abstract}
In 2018, 70\% of the Portuguese companies produced thousands of Portuguese Audit Tax documents (SAF-T (PT)) files for tax validation. These documents represent a standardized procedure for the Portuguese companies, providing the necessary data about billing, accounting, and taxation. These files contain valuable information that can represent an important tool for analytical procedures to support decision-making processes. Thus, a Business Intelligence System based on SAF-T (PT) was created to support companies' analytical needs. An important decision-making process involves customer evaluation. So, the proposed system will begin, in a preliminary phase, with RFM analysis, for customer segmentation using Clustering techniques and results were cross-validated using Decision Tree and Linear Discriminant Analysis. Additionally, a brief interpretation of marketing strategies was suggested and a comparison between Rapid Miner and SPSS was synthesized. The results show that it is possible to explore SAF-T (PT) files to extract knowledge different than tax purposes, namely, the determination of customer profiles. Accuracy is superior to $80 \%$ in both software and techniques.
\end{abstract}

\section{KEYWORDS}

Data Mining, SAF-T (PT), Data Warehousing, Customer Segmentation, ETL

\section{INTRODUCTION}

Since 2017, the Portuguese Tax Authority (PTA) requires all companies that have organized accounting (approx.70\%) to monthly submit the Standard Audit File for Tax Purposes ${ }^{2}$ (SAF-T (PT)) for validation. The SAF-T (PT) documents represent valuable data extracted from the companies' operational systems. Despite having these data, several companies do not have mechanisms to create deeper knowledge from the data they produce. Owning data alone is not enough anymore to get practical results. Data need to make sense and be properly framed and aligned with the organization goals to become useful information for managers, who daily make decisions that affect the organization and consequently the stakeholders. This is nowadays the basis for growing business activities. In this paper, we explore the use of billing SAF-T (PT) documents to create a Business Intelligence (BI) System to support decision-making requirements. These documents store monthly data about several organization entities such as customers, products, and sales. This data can be very useful to provide data insights about the organization's business. Thus, a Data Warehouse (DW) can be generated based on SAF-T (PT) data and used within exploratory techniques to extract important findings about the business domain. We explore the customer perspective for data analyses, revealing how SAF-T (PT) data can be used to provide customer segmentation.

For data exploration, the Recency, Frequency and Monetary analysis (Bult and Wansbeek 1995) is performed. This is a customer equity analysis that companies can perform to evaluate and improve their relationship to this important stakeholder. RFM analysis is based on the following measures: Recency (R), which represents the period since customer's last purchase; Frequency $(\mathrm{F})$, is the number of orders in a certain period and, Monetary (M) which represents the amount that the customer spent in the same interval. To perform the RFM method, we resort to Data Mining (DM) (Han, Kamber, and Pei 2012), more specifically, a clustering

\footnotetext{
\{8180717, bmo, mrc, tsm $\} @$ estg.ipp.pt

${ }^{2}$ https://www.oecd.org/tax/administration/45167181.pdf
} 
technique, for grouping the customers based on its RFM values. DM allows us to extract knowledge of large datasets, which means, discover hidden and unknown patterns (or useful information) from large databases.

The rest of this paper is organized as follows: the second section is dedicated to a brief literature review; section 3 presents the case study and the validation of the results obtained from the different methods and tools and finally, in section 4 conclusions are addressed and future work suggested.

\section{RELATED WORK}

DM is widely used in decision support, forecasting, description and prediction in many application fields, like, banking, fraud detection, customer relationship management, and several other fields (Han, Kamber, and Pei 2012; Deshpande and Thakare 2010).

A review on DM techniques are presented in the work of Chen et al. (F. Chen et al. 2015) and Deshpande and Thakare (Deshpande and Thakare 2010). Both authors classify DM by the method used and their applications, in a specific domain. In both documents, clustering and classification analysis appear frequently. Clustering analysis is an unsupervised method widely used in the literature with several algorithms and techniques (Berkhin 2006). K-means is a widely used clustering technique that intends to group individuals according to its characteristics and use that information to support decision-making processes (Aviad and Roy 2011). In the order hand, classification is a supervised method that aims to create models used for predicting class labels within unknown records. DT and LDA are supervised methods used for classification or clustering validation.

As we are dealing with SAF-T (PT) data, where the document is oriented towards sales facts, we can perform several analyzes regarding the Customer Relationship Management (CRM) as discussed in (Tsiptsis and Chorianopoulos 2010). Introduced by (Bult and Wansbeek 1995), RFM is implemented to quantify customer equity and to focus on relationship management efforts. Later several approaches were carried out, for instance, weighted RFM in which weights are assigned to each variable according to business characteristics (Zaheri et al. 2012) and augmented RFM, where other variables are added, such as relationship duration, churn probability (Chan, Cheng, and Hsien 2011) or Count item (Khajvand et al. 2011). On the other hand, Chen et al. (Y. L. Chen et al. 2009) were the first to apply the RFM criterion in sequential patterns and, finally, Chan (Chan, Cheng, and Hsien 2011) combined RFM and prices optimization for on-line customers to personalize promotions and allow clients to bargain the conceded prices.

In several studies, thresholds are being specified by domain experts as shown in (Marcus 1998), (Khajvand et al. 2011) or in (Sohrabi and Khanlari 2007). In these papers, authors grouped individuals based on particular limits for each variable and then calculate the correspondent customer life value (CLV), as an index of importance for the company.

In this study, we do not intend to order or rank customers, but to distinguish and value each group of this special stakeholder. In the present study, clustering methods are used for "natural" customer segmentation instead of forcing customers into groups using specified thresholds. The objective is to identify customer characteristics that allow differentiating the company's response with appropriate marketing strategies. We consider that the analysis of natural clusters can help decision-makers identifying pattern clearly and associate market segments easily without partitioning customers a priori and, consequently, develop more effective strategies, for instance, targeted marketing (Marcus 1998).

\section{RFM ANALYSIS}

Even with limited organizational data (only billing and accounting business processes are covered), the SAF-T (PT) documents can represent the foundations to provide analytical procedures to support decision-making processes. This kind of standard data representation can be used to create a Data Warehousing System (DWS) as BI integral component, or, at least, a skeleton that can be used to ground the application of advanced DM techniques and the application of typical analytical procedures to support decision-making (considering perspectives presented in the billing SAF-T (PT) documents such as customer, products or suppliers). 
However, even considering a standard data representation for SAF-T (PT), several problems can occur on data instances used to create each document. While structural constraints can be easily validated (using the correspondent XML Schema), the integrity of these data can be difficult to guarantee due to data entry errors or data changes framed on a specific time window. Additionally, history maintenance cannot be guaranteed using only SAF-T (PT) files because of each file stores self-contained data about customers, products, tax values, and invoices for a specific period. When analytical procedures are applied, data lineage and evolution are compromised because we have a small picture of the organization at a specific point in time. With a DW system, data that change over time can be captured, providing properly framed data states that can allow for deep data analysis.

We idealized a simple DW that captures sales events using a fact table and a set of dimension tables proving context and different perspectives for sales data analysis. The Sales fact table represents a sale line event with several measures. The amount is one of them, representing the Sale line amount obtained by the formula: Quantity * UnitPrice. Additionally, the DW schema comprises six dimensions: The temporal data dimension (Calendar); the Customer dimension, represented by the identification of the customer, type and an address hierarchy: City -> Region -> Country -> ALL; the Invoice dimension in which each fact belongs; the Product dimension; Tax dimension providing context about each sale; and the Company dimension holding the sales data. This is just an example of a possible configuration. Additional business processes could be included in the DW configuration. For example, the "movement of goods" related data could be a good candidate to extend the DW applicability.

In this study, we use this DW and propose a methodology (Figure 1) to perform the customer segmentation analysis and the validation of the outcome. First, it starts with the RFM analysis. A clustering algorithm is applied to the dataset in order to identify clusters according to the customers' characteristics (RFM variables). The first stage of the clustering algorithm implies to pre-process the available data. The second stage of the methodology consists of performing the clustering analysis (modelling phase), and it is previously required to determine the number of $k$ clusters. After the modelling phase, the validation of the outcome of the clustering algorithm is performed, since the created clusters must be validated using supervised learning methods (Marôco, (2018)). So, DT and LDA are both used to validate the clustering outcome.

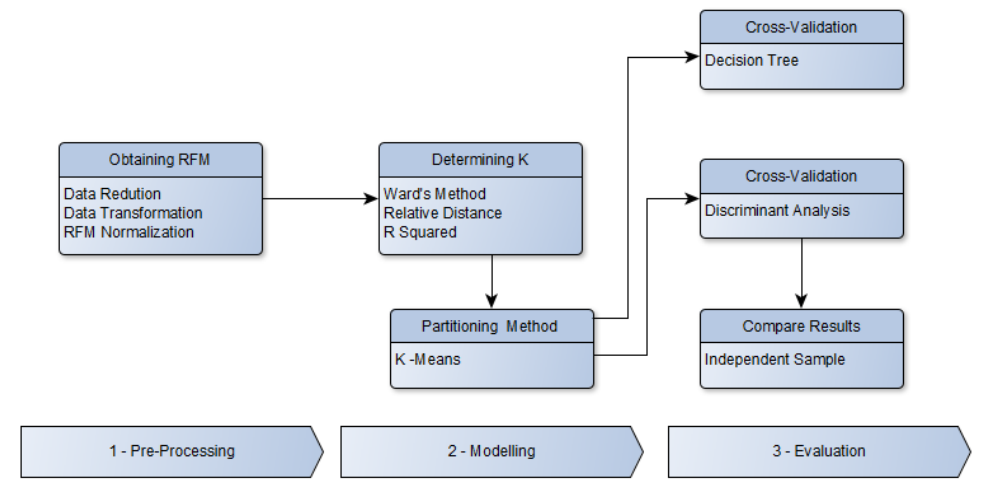

Figure 1. RFM analysis approach

To illustrate the proposed methodology, we randomly choose a company from the dataset. The following 5 attributes (from the original 84) were selected: SKCompany, CustomerID, SKInvoice; InvoiceDate and Amount. In the data pre-processing phase, the data is prepared and transformed into the application of the algorithm. The SKCompany attribute represents a particular company; the CustomerID is used to aggregate invoices with the right customer; using InvoiceDate we generate the new variable Recency by calculating the difference between the date of the last purchase with the actual one; the Frequency is a new attribute derived by the count of SKInvoices and finally to obtain Monetary the average purchase amount for one year was used. Total Monetary and Frequency trends to be correlated, hence the total amount spent in the same period was replaced by the average as proposed by (Marcus 1998). For illustrative purposes, we only perform the RFM analysis on the SKCompany number 8501 with 620 product lines, 124 invoices and 49 customers over one year. As shown in Table 1, in average, the period since a customer last purchase is 9.2 months, the number of purchases made within a year is approximately 13 and a customer usually spends about 4885 euros per year. 
Table 1. Descriptive Statistics (SPSS)

\begin{tabular}{|lr|r|r|r|r|}
\hline \multicolumn{7}{c|}{ Descriptive Statistics } \\
& N & Minimum & Maximum & \multicolumn{1}{c|}{ Mean } & Std. Deviation \\
\hline Monetary & 49 & 2920,20 & 7518,00 & 4885,3594 & 938,79433 \\
\hline Recency & 49 & 4,99 & 16,46 & 9,2063 & 3,18958 \\
\hline Frequency & 49 & 5 & 30 & 12,65 & 7,507 \\
\hline Valid N (listwise) & 49 & & & & \\
\hline
\end{tabular}

\subsection{Determining the Best $k$}

Neither Rapidminer ${ }^{3}$ nor SPSS ${ }^{4}$ provides satisfactory indexes to assess the best number of clusters to be chosen to perform the k-means algorithm. Therefore, both distance between clusters and $R^{2}$ criteria will be used to decide the final number of clusters. $R^{2}$ represents the proportion of the total variability that is retained by the factor for all $p$-variables and $k$ solutions and can be processed by applying three one-way ANOVA and comparing the sum of squares within groups with the sum of squares total. For both criteria, the square Euclidean distance (Johnson, Wichern, and others 2002) and the Ward's Method (Anderberg 1973) were used in SPSS, once Rapidminer does not have similar output. On the other hand, from the SPSS outputs, namely from the "Agglomeration Schedule", it is possible to select the last agglomerations and describe relative distances between clusters as shown in Figure 2.

As the number of clusters increases, $R^{2}$ increases since it accumulates more variability. On the contrary, as the groups aggregate, the relative distance between them decreases, until the final condition in which all objects are in the same final cluster, where the distance is zero.

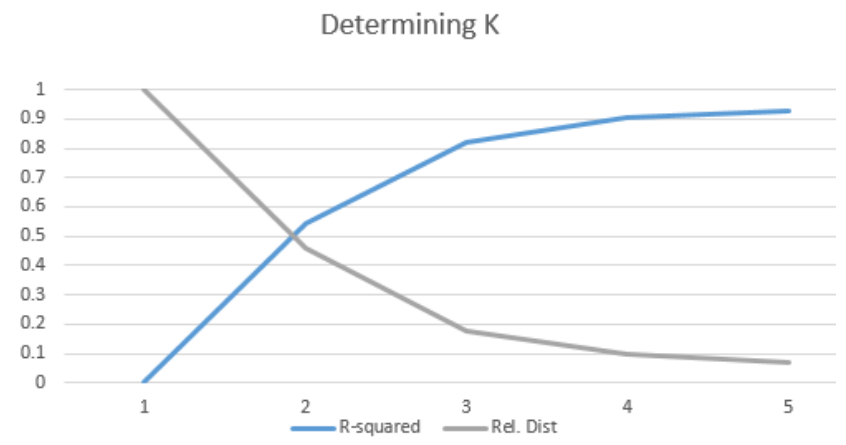

Figure 2. Relative distance and R squared

From the relative distance criteria, the best $k$ is identified by the most pronounced "elbow" that is formed on the downward curve. This criterion is subjective, but in this case, it is visually clear that $k=3$.

The $R^{2}$ criterion is more objective once we can determine that the best solution for $\mathrm{k}$ is the smallest number of clusters that retains a significant percentage of the total variability, let us say $R^{2}=80 \%$. Then, the number of clusters satisfying those conditions is $k=3$.

Once we have determined the number of clusters, the classification of each subject in the retained clusters may be refined with the non-hierarchical k-means procedure as follows.

\footnotetext{
${ }^{3}$ https://rapidminer.com/

${ }^{4}$ https://www.ibm.com/analytics/spss-statistics-software
} 


\subsection{K-means Application}

While hierarchical algorithms gradually (dis)assemble objects into clusters, partitioning methods calculate distances based on the pre-defined 3 clusters. The output that is generated by each tool is the point in the center of each cluster - the centroids. Then, by centroids coordinates knowledge can be interpreted.

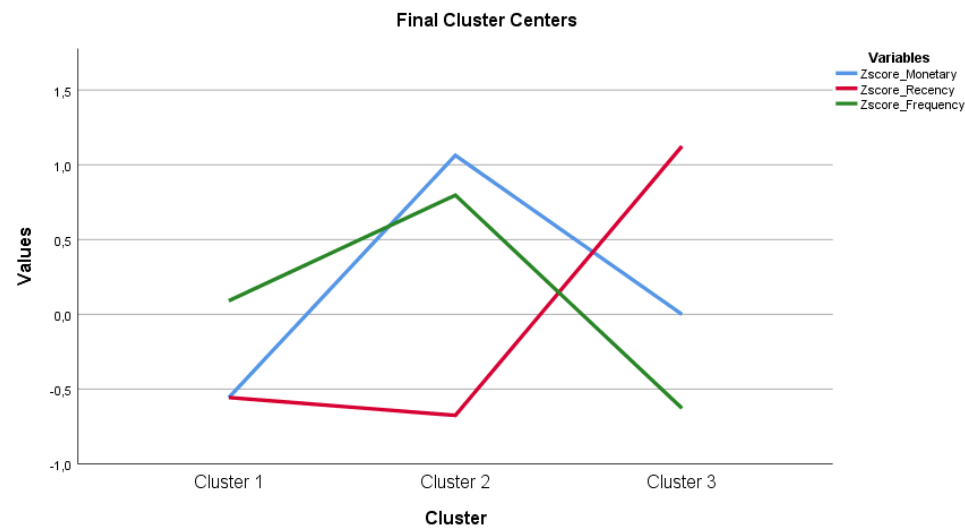

Figure 3. K-means centroids coordinates from customers (SPSS)

We can extract and interpret knowledge from the coordinates of the centroids shown in Figure 3 . There are two centroids with the opposite behaviors, 2 and 3. While the type of customers represented in cluster 2 can be considered as a "heavy spender" (with high monetary values), its last purchase was very recent (low recency values) and frequently make purchases (high-frequency values), the type of customers represented in cluster 3 may be interpreted as a "minor spender" (with low monetary values), an infrequent customer (low-frequency values), and does not do purchases for a long time (high recency values). Finally, the type of customer represented in cluster 1 is a "light spender" (with low monetary values, in average), visits frequently the company (high-frequency values) and placed orders recently (low recency values).

The centroids analysis yields insights into marketing strategies and allows to address specific products, campaigns and optimize marketing costs. For instance, the customer in cluster 2 could be classified as an "easy customer", the one in cluster 3 "aversion to shopping" and finally customer in cluster 1 "adhering to promotions".

\subsection{Validation of the K-means Outcome}

According to (Marôco, 2018), the clusters formed by this heuristic method must be, à posteriori, validated using supervised learning methods. Both Rapidminer and SPSS produce a confusion matrix, which can be analyzed and interpreted and to consequently validate the k-means model performance. In both cases, the leave-one-out cross-validation is performed. This is a process in which one observation is left out of the sample then the model is redefined by the other cases and, finally, each case is reclassified by the new model including the one that was kept out.

\subsubsection{Validation with Decision Tree}

A DT algorithm generates a tree structure, where each internal node denotes a test on an attribute, each branch corresponds to an outcome of the test and each leaf holds a class label (Han, Kamber, and Pei 2012).

After performing the DT algorithm in our dataset, a tree structure is created. This structure allows to deduce the variable that best separates the groups is Recency, which defines high or low recency customers. Then, another decision is applied in the Frequency variable. These two variables are the main responsible for the partition of the groups by this method. Overall, both values are quite high: SPSS presents the higher accuracy value with $87,8 \%$, while Rapidminer presents $85,7 \%$, as shown in table below (Table 2). 
Table 2. Validation using DT (Rapidminer and SPSS)

Validation by DT - Rapidminer

\begin{tabular}{|l|l|l|l|l|}
\hline & $\begin{array}{l}\text { true } \\
\text { cluster_1 }\end{array}$ & $\begin{array}{l}\text { true } \\
\text { cluster_2 }\end{array}$ & $\begin{array}{l}\text { true } \\
\text { cluster_0 }\end{array}$ & $\begin{array}{l}\text { class } \\
\text { precision }\end{array}$ \\
\hline pred. cluster_1 & 12 & 0 & 0 & $100.0 \%$ \\
\hline pred. cluster_2 & 3 & 26 & 3 & $81.3 \%$ \\
\hline pred. cluster_0 & 0 & 1 & 4 & $80.0 \%$ \\
\hline class recall & $80.0 \%$ & $96.3 \%$ & $57.1 \%$ & $85.7 \%$ \\
\hline
\end{tabular}

Validation by DT - SPSS

\begin{tabular}{|l|r|l|l|l|}
\hline & $\begin{array}{l}\text { true } \\
\text { cluster_1 }\end{array}$ & $\begin{array}{l}\text { true } \\
\text { cluster_2 }\end{array}$ & $\begin{array}{l}\text { true } \\
\text { cluster_0 } 0\end{array}$ & $\begin{array}{l}\text { class } \\
\text { precision }\end{array}$ \\
\hline pred. cluster_1 & 20 & 2 & 1 & $87.0 \%$ \\
\hline pred. cluster_2 & 1 & 9 & 2 & $75.0 \%$ \\
\hline pred. cluster_0 & 0 & 0 & 14 & $100.0 \%$ \\
\hline class recall & $95.2 \%$ & $81.8 \%$ & $82.4 \%$ & $87.8 \%$ \\
\hline
\end{tabular}

\subsubsection{Validation with Linear Discriminant Analysis}

The LDA can be performed by various methods. It is a very robust technique against the violation of assumptions although all assumptions were tested and confirmed. Wilks' lambda statistic measures the discriminant power of a variable; however, it is only valid if the variables are independent and if the samples come from a normal multivariate population whose variability is homogeneous in each group.

From the stepwise method, all the variables proved to be significantly important for differentiating groups ( $\mathrm{p}$-value $<0.001)$. Recency was the first to enter with the minor value compared with the other variables in step $1\left(\Lambda_{R}=0.312\right)$. In step2, considering the remain variance, Monetary was chosen against Frequency $\left(\Lambda_{M}=0.189\right)$ and lastly Frequency joined the set in step $3\left(\Lambda_{F}=0.150\right)$. However, we are still unable to identify which clusters are distinguished from each variable. Therefore, two of Fisher's Discriminant Functions (FDF) were extracted based on the canonical correlation between discriminant functions and groups. Table 3 presents the standardized coefficients of each RFM variable in the discriminating functions 1 and 2 , the percentage of explained variances based on the eigenvalues and finally the statistics and the corresponding $\mathrm{p}$-value. Note that each function is highly significant in discriminating groups $(\mathrm{p}-$ value $<0.001)$ however, the power of the first is vastly superior than the second once the percentage of variance retained is $76 \%$ against $24 \%$, respectively.

Table 3. Discriminant Functions coefficients and statistics (adapted from SPSS output)

\begin{tabular}{|l|r|r|r|r|r|r|r|}
\hline \multicolumn{10}{|c|}{ Standardized Coefficients in Discriminant Function } \\
\hline Function & Recency & Frequency & Monetary & Eigenvalue & $\%$ of Variance & Wilk's Lambda & \multicolumn{1}{c|}{$p$-value } \\
\hline 1 & 0.899 & -0.437 & -0.235 & 2,633 & 75.886 & 0.150 & $<0.001$ \\
2 & 0.312 & 0.427 & 0.957 & 0,837 & 24.114 & 0.554 & $<0.001$ \\
\hline \multicolumn{10}{|c|}{} \\
\cline { 1 - 7 }
\end{tabular}

The structural coefficients were also considered but, since the correlation between variables and functions are weak, we obtained similar values, so we kept the standardized coefficients. Additionally, the first function is essentially defined by the variable Recency $(0,899)$ and the second by Monetary $(0,957)$. Thus, Figure 4 illustrates the positioning of each customer on the territorial map obtained by the scores of the FDF whose most significant variable is associated.

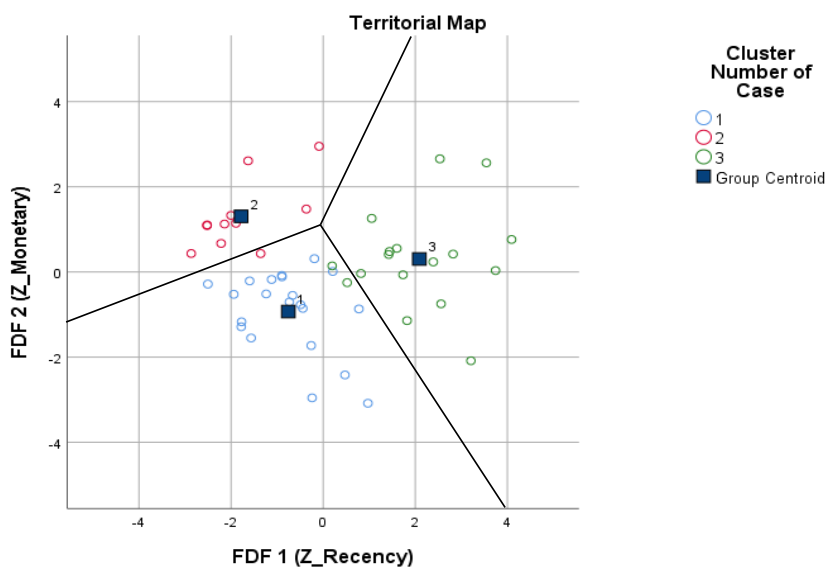

Figure 4. Territorial Map (adapted from SPSS output) 
The black lines are the borders and were added manually since the SPSS territorial map is very rudimentary. Note that one instance classified in cluster 2 (in red) by K-means was classified by LDA in the first group and two individuals from cluster 3 fell into the predicted group 1. Using the interpretation of the clusters of Figure 4 , the outcome of both tools of the application of the LDA algorithm and the composition of the final clusters represented in Table 4, we can conclude that the accuracy values are better but very similar to the ones in the DT validation approach. All values are quite high, over $83 \%$. SPSS presents a higher accuracy value with $91,8 \%$, while Rapidminer presents $85,7 \%$ accuracy value.

Table 4. Validation using LDA (Rapidminer and SPSS)

\begin{tabular}{|c|c|c|c|c|c|c|c|c|c|}
\hline \multicolumn{5}{|c|}{ Validation by LDA - Rapidminer } & \multicolumn{5}{|c|}{ Validation by LDA - SPSS } \\
\hline & \begin{tabular}{|l} 
true \\
cluster_1
\end{tabular} & $\begin{array}{l}\text { true } \\
\text { cluster_2 }\end{array}$ & $\begin{array}{l}\text { true } \\
\text { cluster_0 }\end{array}$ & $\begin{array}{l}\text { class } \\
\text { precision }\end{array}$ & & \begin{tabular}{|l} 
true \\
cluster_1
\end{tabular} & $\begin{array}{l}\text { true } \\
\text { cluster_2 }\end{array}$ & $\begin{array}{l}\text { true } \\
\text { cluster_0 }\end{array}$ & \begin{tabular}{|l} 
class \\
precision
\end{tabular} \\
\hline pred. cluster_1 & 12 & 0 & 0 & $100.0 \%$ & pred. cluster_1 & 20 & 1 & 2 & $87.0 \%$ \\
\hline pred. cluster_2 & 3 & 27 & 4 & $79.4 \%$ & pred. cluster_2 & 1 & 10 & 0 & $90.9 \%$ \\
\hline pred. cluster_0 & 0 & 0 & 3 & $100.0 \%$ & pred. cluster_0 & 0 & 0 & 15 & $100.0 \%$ \\
\hline class recall & $80.0 \%$ & $100.0 \%$ & $42.9 \%$ & $85.7 \%$ & class recall & $95.2 \%$ & $90.9 \%$ & $88.2 \%$ & $91.8 \%$ \\
\hline
\end{tabular}

\subsubsection{Independent Sample}

Additionally, we applied the same methodology process in another randomly chosen company. Company 8585 contains information about 47 clients, 168 invoices from Jan 1, 2019 to Dec 31, 2019. The results were even better, compared with 8501 's results (Table 5).

Table 5. Comparison of Accuracy from different techniques and tool

\begin{tabular}{|c|c|c|c|c|c|}
\hline \multicolumn{3}{|c|}{ Company 8501, Accuracy } & \multicolumn{3}{|c|}{ Company 8585, Accuracy } \\
\hline & D. T. & L. D. A. & & D. T. & L. D. A. \\
\hline RM & 85.7 & 85.7 & RM & 87.2 & 95.7 \\
\hline SPSS & 87.8 & 91.8 & SPSS & 83 & 95.7 \\
\hline
\end{tabular}

In both cases, in DT and LDA, accuracy values are also high (above 83\%). These numbers lead us to look with optimism for a future validation with real data where, generally, the number of invoices is higher as also the number of customers. If these conditions are satisfied, according to the Central Limit Theorem, the approximation of distributions within groups to normal should be significant and the discriminating power of variables and functions should be higher what leads us to expected higher levels of accuracy for LDA.

\section{CONCLUSIONS}

In this work, we perform a customer segmentation analysis using the RFM criterion considering SAF-T (PT) data properly structured in a DW repository oriented to support selective strategies for sales. To perform the customer analysis, we apply a clustering technique and the consequent results were validated using supervised techniques, Decision Tree and Linear Discriminant Analysis.

The proposed methodology started with the pre-processing phase, which is essential to prepare the dataset to the application of the clustering technique. In the modelling phase, we applied the k-means algorithm to the dataset and afterwards, we used the leave-one-out cross-validation using two supervised methods. After the validation phase and interpreting the results, we obtained accuracy values greater than $83 \%$ in both tools and both algorithms. Concerning RFM analysis, a small number of clusters was determined, which facilitates interpretation. Understanding the characteristics of each group makes it possible to establish a relationship with customers to facilitate the direction of marketing strategies. Still, we would like to repeat this analysis with additional variables like geographic, demographic or customer preferences for future work.

From another perspective, data from real companies must be tested, for example, to test, if the position of a company in the distribution chain may affect the results, as well as the capacity for on-line business. The validations by DT and LDA from both tools obtained high levels of accuracy (above 83\%). The best result was achieved by the LDA method in both programs, so this procedure should be considered for the classification and validation of clusters. It is intended to test the results with the application of Neural Network or other 
techniques for the validation of the clustering model. As for the dataset, it is expected that the application to other companies will produce also high levels of accuracy.

The RFM analysis has shown to be effective in the SAF-T (PT) dataset. It is suggested that the application of these techniques into real data could produce essential knowledge to decision-makers, helping them to drive their companies further into an increasingly competitive market. These findings are interesting since they prove the effectiveness of a self-service BI system that can be generated based on SAF-T (PT) data and possibly extended by companies' specific data. Thus, mainly the small companies can use it as a faster and cheaper way to initiate their BI system to support their tactical and strategic decisions. As future work, we intend to exploit this approach for BI implementation.

\section{ACKNOWLEDGMENT}

This work has been supported by national funds through FCT - Fundação para a Ciência e Tecnologia through project UIDB/04728/2020.

\section{REFERENCES}

Anderberg, M. R. 1973. Cluster Analysis for Applications. New York: Academic press.

Aviad, Barak, and Gelbard Roy. 2011. "Classification by Clustering Decision Tree-like Classifier Based on Adjusted Clusters." Expert Systems with Applications 38 (7): 8220-28.

Berkhin, P. 2006. "A Survey of Clustering Data Mining Techniques BT - Grouping Multidimensional Data.” Grouping Multidimensional Data, no. c: 25-71.

Bult, J.R., and T.J. Wansbeek. 1995. “Optimal Selection for Direct Mail.” Marketing Science 14(4): 378-95.

Chan, C. C.Henry, Chi Bin Cheng, and Wen Chen Hsien. 2011. "Pricing and Promotion Strategies of an Online Shop Based on Customer Segmentation and Multiple Objective Decision Making." Expert Systems with Applications 38 (12): 14585-91.

Chen, Feng, Pan Deng, Jiafu Wan, Daqiang Zhang, Athanasios V. Vasilakos, and Xiaohui Rong. 2015. "Data Mining for the Internet of Things: Literature Review and Challenges." International Journal of Distributed Sensor Networks 2015 (i).

Chen, Yen Liang, Mi Hao Kuo, Shin Yi Wu, and Kwei Tang. 2009. "Discovering Recency, Frequency, and Monetary (RFM) Sequential Patterns from Customers' Purchasing Data.” Electronic Commerce Research and Applications 8 (5): 241-51. https://doi.org/10.1016/j.elerap.2009.03.002.

Deshpande, S.P, and V.M Thakare. 2010. "Data Mining System and Applications: A Review." International Journal of Distributed and Parallel Systems 1 (1): 32-44.

Han, Jiawei, Micheline Kamber, and Jian Pei. 2012. Data Mining: Concepts and Techniques. Morgan Kaufmann Publishers. Third Edit. Morgan Kaufmann Series.

Johnson, Richard Arnold, Dean W Wichern, and others. 2002. Applied Multivariate Statistical Analysis. Vol. 5. Prentice hall Upper Saddle River, NJ.

Khajvand, Mahboubeh, Kiyana Zolfaghar, Sarah Ashoori, and Somayeh Alizadeh. 2011. "Estimating Customer Lifetime Value Based on RFM Analysis of Customer Purchase Behavior: Case Study." Procedia Computer Science 3: 57-63.

Marcus, Claudio. 1998. "A Practical yet Meaningful Approach to Customer Segmentation." Journal of Consumer Marketing 15 (5): 494-504.

Marôco, João. 2018. Análise Estatística Com Utilizaçao Do SPSS 25. Edições Sílabo, Lisboa. 7th ed.

Sohrabi, Babak, and Amir Khanlari. 2007. "Customer Lifetime Value (CLV) Measurement Based on RFM Model." Iranian Accounting \& Auditing Review 14 (47): 7-20.

Tsiptsis, Konstantinos, and Antonios Chorianopoulos. 2010. Data Mining Techniques in CRM: Inside Customer Segmentation. Data Mining Techniques in CRM: Inside Customer Segmentation. https://doi.org/10.1002/9780470685815.

Zaheri, Fayegh, Hiwa Farughi, Hersh Soltanpanah, Seiran Alaniazar, and Foruzan Naseri. 2012. "Using Multiple Criteria Decision Making Models for Ranking Customers of Bank Network Based on Loyalty Properties in Weighted RFM Model.” Management Science Letters 2 (2): 697-704. 